\title{
Local active black hole mass functions
}

\author{
Jenny E. Greene ${ }^{1}$ and Luis C. Ho ${ }^{2}$ \\ ${ }^{1}$ Department of Astronomy, Princeton University, Princeton, NJ 08544, USA \\ 2 The Observatories of the Carnegie Institution of Washington, Pasadena, CA 91101, USA \\ email: jgreene@astro.princeton.edu,lho@ociw.edu
}

\begin{abstract}
While black holes (BHs) are apparently a ubiquitous component of the nuclei of local spheroids, their role in galaxy evolution remains largely unknown. The tight correlations between galaxy spheroid properties and $\mathrm{BH}$ mass provide an important boundary condition for models of the coevolution of BHs and galaxies. Here we consider another important boundary condition: the local mass function of broad-line active galaxies. We use standard virial mass estimation techniques to examine the distribution of $\mathrm{BH}$ masses and accretion rates for active galaxies in the local universe, and we also compare the distribution of $\mathrm{BH}$ masses in local broad and narrow-line objects, and find that both populations have a characteristic mass of $\sim 10^{7} M_{\odot}$. Most importantly, this is the first BH mass function to consider BH with masses $<10^{6} M_{\odot}$. The space density of this important population allows us to place constraints on potential mechanisms for the creation of seed BHs in the early Universe.
\end{abstract}

Keywords. galaxies: active - galaxies: Seyfert - galaxies: evolution

\section{Introduction}

The distribution of black hole $(\mathrm{BH})$ mass density in the local Universe provides an important boundary condition on the cosmological growth of BHs. Because of tight scaling relations between $\mathrm{BH}$ mass $\left(M_{\mathrm{BH}}\right)$ and spheroid properties, including luminosity (e.g., Marconi \& Hunt 2003) and stellar velocity dispersion (the $M_{\mathrm{BH}}-\sigma_{*}$ relation; Tremaine et al. 2002) it is possible to convert observed luminosity or $\sigma_{*}$ functions into BH mass density, and thus constrain the primary growth modes of supermassive BHs (e.g., Yu \& Tremaine 2002; Marconi et al. 2004). There is also useful information to be gleaned by looking at the masses and Eddington ratios of currently growing (i.e., active) BHs. Heckman et al. (2004) showed that while the characteristic BH mass for all BHs locally is $\sim 10^{8} M_{\odot}$, narrow-line active galactic nuclei (AGNs) from the SDSS have significantly lower masses, $\sim 3 \times 10^{7} M_{\odot}$, where $M_{\mathrm{BH}}$ is inferred from $\sigma_{*}$, assuming the $M_{\mathrm{BH}}-\sigma_{*}$ relation holds. Here, we present a complementary study based on broad-line AGNs, for which it is possible to derive BH masses using scaling relations between AGN luminosity and broad-line region size (e.g., Kaspi et al. 2005; Greene \& Ho 2005). Amongst other things, this sample allows us to look at $M_{\mathrm{BH}}$ without direct dependence on the $M_{\mathrm{BH}}-\sigma_{*}$ relation, directly compare the mass distribution of broad and narrow-line AGNs, and, in particular, probe the mass regime $M_{\mathrm{BH}} \lesssim 10^{6} M_{\odot}$. Although such low-mass BHs contribute a negligible fraction of both the total BH mass density and the integrated AGN luminosity density, their space density provide important constraints on the mass distribution of primordial seed BHs (e.g., Haehnelt 2004) and source counts for gravitational wave experiments such as LISA (e.g., Hughes 2002). Because of spectral resolution limitations, current mass distributions inferred from $\sigma_{*}$ do not probe below $3 \times 10^{6} M_{\odot}$, while we do not know how to convert galaxy luminosity into BH mass for low-mass galaxies. 

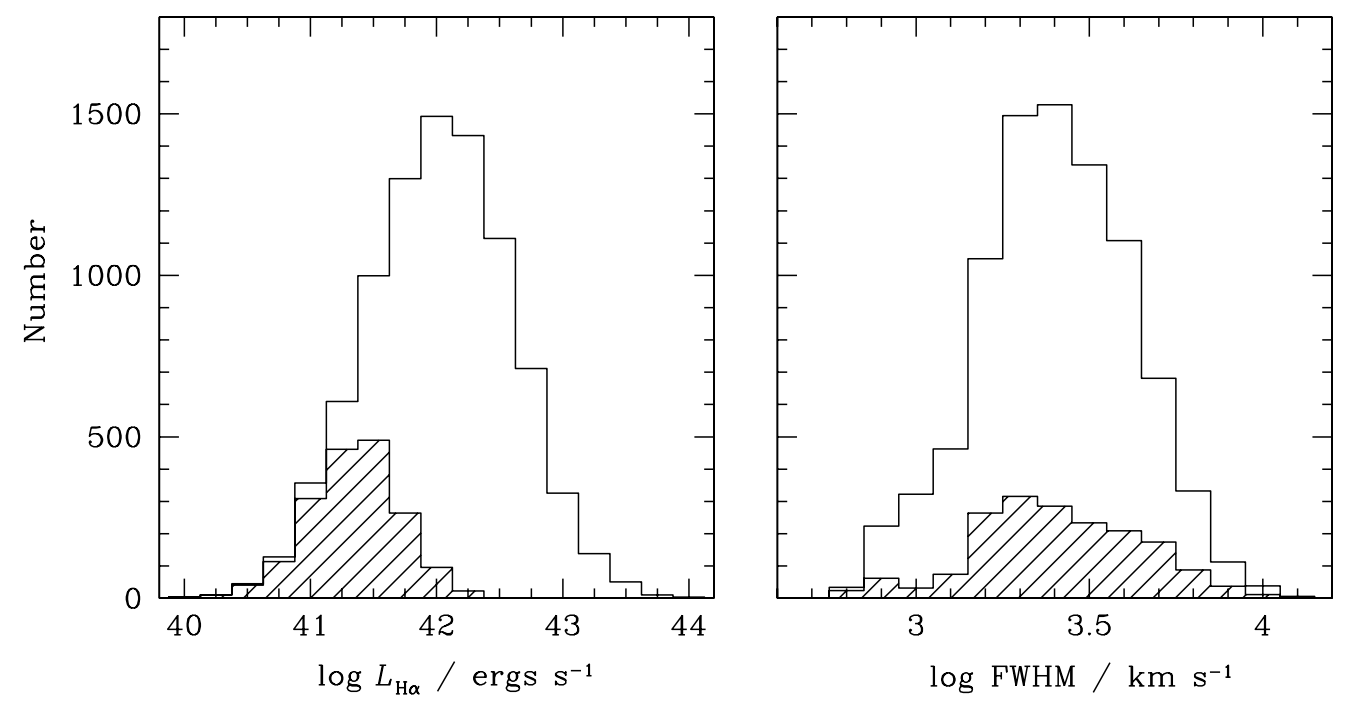

Figure 1. Distributions of broad $L_{\mathrm{H} \alpha}$ (left) and broad $\mathrm{FWHM}_{\mathrm{H} \alpha}$ (right) for the entire sample of broad-line AGNs. The shaded histograms indicate the distribution of the sample targeted by the SDSS main galaxy sample. Note the dominance of objects with $\mathrm{FWHM}_{\mathrm{H} \alpha}$ below the canonical division at $\mathrm{FWHM}_{\mathrm{H} \alpha}=2000 \mathrm{~km} \mathrm{~s}^{-1}$, and that while the galaxy selected AGNs are uniformly at low luminosities, they span the entire range of $M_{\mathrm{BH}}$ in the sample. Massive BHs are predominantly in low accretion states at the present time.

\section{Results}

We follow the procedure outlined in Greene \& Ho (2004) using the Fourth Data Release of SDSS (Adelman-McCarthy et al. 2006). Briefly, galaxy continuum is modeled and removed using the principal component analysis method of Hao et al. (2005), narrow $\mathrm{H} \alpha+\left[\mathrm{N}_{\mathrm{II}}\right] \lambda \lambda 6548,6583$ are modeled and removed using a model of the [SII] $\lambda \lambda 6716,6731$ lines, and finally, when necessary, broad $\mathrm{H} \alpha$ is modeled as the sum of Gaussian components. The resulting sample of $\sim 8300$ AGNs have the distribution of $\mathrm{FWHM}_{\mathrm{H} \alpha}$ and $L_{\mathrm{H} \alpha}$ shown in Figure 1. Using the scaling relations presented in Greene \& Ho (2005), we find a raw distribution of $M_{\mathrm{BH}}$ and $L_{\mathrm{bol}} / L_{\mathrm{Edd}}$ shown in Figure 2.

We use the classic $V / V_{\max }$ method to compute the broad $\mathrm{H} \alpha$ luminosity function. The maximum volume to which we would detect a source depends both on its total luminosity (it must be spectroscopically targeted by the SDSS) and its $\mathrm{H} \alpha$ luminosity and signalto-noise ratio $(\mathrm{S} / \mathrm{N}$; our algorithms must detect the object as a broad-line AGN). [Note that we include objects in our sample that were targeted both as galaxies (limiting magnitude $r<17.77)$ and quasars $(i<19.1)$, which slightly complicates the analysis.] To estimate the limit of our ability to recognize broad $\mathrm{H} \alpha$ we create model spectra at a range of redshifts and perform our entire selection procedure on each object. The final luminosity function is shown as filled circles in Figure 3, while the solid line shows our maximal luminosity function, including objects with AGN-like line ratios, but weak $\mathrm{H} \alpha$ emission for which simulations show we may not reliably measure a $\mathrm{BH}$ mass. We are clearly incomplete below a few $\times 10^{40} \mathrm{ergs} \mathrm{s}^{-1}$; the Palomar survey, which by virtue of its smaller aperture and much brighter magnitude limit is sensitive to much lower $\mathrm{H} \alpha$ luminosities, continues to rise toward lower luminosity than probed by this study (Ho 2004). Nevertheless, we convert the observed luminosity function into a BH mass function using the $V_{\max }$ weights derived above. We find, in agreement with Heckman et al. (2004), that the characteristic mass for this sample is $\sim 10^{7} M_{\odot}$, which is significantly lower than 



Figure 2. Distributions of BH mass (left) and $L_{\mathrm{bol}} / L_{\mathrm{Edd}}($ right $)$ for the entire sample of broadline AGNs. $M_{\mathrm{BH}}$ is calculated from the $L_{\mathrm{H} \alpha}$ and $\mathrm{FWHM}_{\mathrm{H} \alpha}$ shown above, using the formalism of Greene \& Ho (2005). The Eddington ratio is derived assuming an average bolometric correction of $L_{\mathrm{bol}}=9 L_{5100} \AA$. As in the previous figure, the galaxies targeted in the SDSS main sample are indicated with the shaded histogram.

that of inactive BHs. However, because we are sensitive to lower masses than Heckman et al. we find a flat mass function between $10^{6}-10^{7} M_{\odot}$, before the mass function turns over. Our goal is to determine the extent to which the mass function truly turns over below $10^{6} M_{\odot}$, and the extent to which we are simply incomplete.

To investigate the impact of our incompleteness, we run Monte Carlo simulations of BHs with masses from $10^{4}-10^{9.5} M_{\odot}$, Eddington ratios from 0.01-1, and redshifts up to $z=0.352$. Each $\mathrm{BH}$ is assigned a galaxy luminosity using either the $M_{\mathrm{BH}}-L_{\mathrm{bulge}}$ relation of Marconi \& Hunt (2003) at high BH mass, or the $M_{\mathrm{BH}}-\sigma_{*}$ relation combined with the $\sigma_{*}-v_{c}$ relation of Pizzella et al. (2005) and the Tully-Fisher relation of Masters et al. (2006) at low BH mass; observed scatter is included for all relations. Artificial spectra are created for each $\mathrm{BH}$, using a range of galaxy types, AGN spectral shapes and narrow-line strengths. Realistic $\mathrm{S} / \mathrm{N}$ ratios are assigned based on empirical relations derived from the SDSS spectra. We then attempt to detect these artificial galaxies, and derive completeness fractions as a function of mass, Eddington ratio and redshift. At low redshift $(z \lesssim 0.02)$ and high Eddington ratio $\left(0.3<L_{\mathrm{bol}} / L_{\mathrm{Edd}}<1\right)$ we expect to be $\sim 75 \%$ complete for BHs with masses of $10^{5} M_{\odot}$. Therefore, we tentatively conclude that we have detected a real decrease in the space density of BHs with masses $<10^{6} M_{\odot}$, unless BHs in this mass range are preferentially inactive compared to their more massive cousins.

\section{Summary}

We have constructed a BH mass function for local broad-line AGNs, sensitive, for the first time, to BHs with masses $<10^{6} M_{\odot}$. We find that active BHs have generally lower masses than inactive BHs at the present day, in agreement with results for narrow-line objects. Furthermore, we find tentative evidence for a decline in space density or activity below $10^{6} M_{\odot}$. 

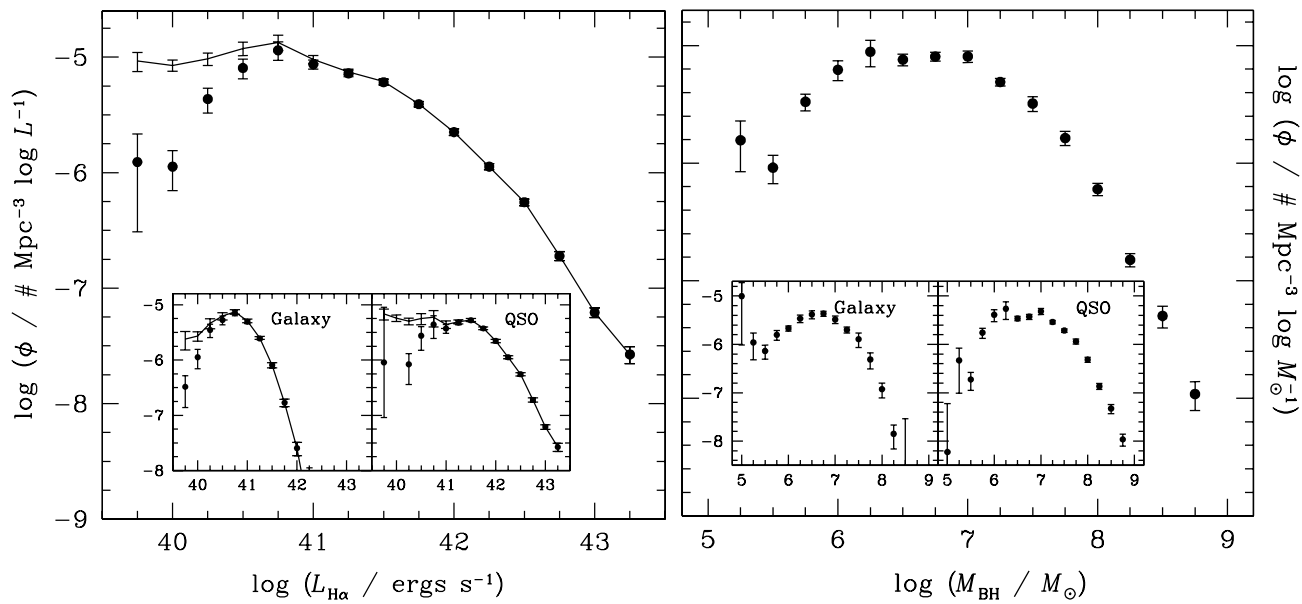

Figure 3. Left: Volume-weighted broad $\mathrm{H} \alpha$ luminosity function in bins of $0.25 \mathrm{dex}\left(\# \mathrm{Mpc}^{-3} \log \right.$ $L_{\mathrm{H} \alpha}{ }^{-1}$ ). The maximum volume is calculated based on both the photometric and spectroscopic limits of the survey and our search algorithm (see text). The error bars represent the Poisson errors in each bin. The inset panels show the luminosity functions for objects targeted as galaxies or quasars, respectively, primarily based on a color selection. Right: Volume-weighted BH mass function in bins of $0.25 \mathrm{dex}\left(\# \mathrm{Mpc}^{-3} \log M_{\mathrm{BH}}{ }^{-1}\right)$. The weights used are identical to those for the luminosity function.

\section{Acknowledgements}

J. E. G. acknowledges an international travel grant from the AAS.

\section{References}

Adelman-McCarthy J. K. et al. 2006, ApJS, 162, 38

Greene J. E., Ho L. C. 2005, ApJ, 630, 122

- 2004, ApJ, 610, 722

Haehnelt M. G. 2004, in Carnegie Observatories Astrophysics Series, Vol. 1: Coevolution of Black Holes and Galaxies, ed. L. C. Ho (Cambridge: Cambridge Univ. Press), 405

Hao L. et al. 2005, AJ, 129, 1783

Heckman T. M., Kauffmann G., Brinchmann J., Charlot S., Tremonti C. \& White, S. D. M. 2004, ApJ, 613, 109

Ho L. C. 2004, in Carnegie Observatories Astrophysics Series, Vol. 1: Coevolution of Black Holes and Galaxies, ed. L. C. Ho (Cambridge: Cambridge Univ. Press), 292

Hughes S. A. 2002, MNRAS, 331, 805

Kaspi S., Maoz D., Netzer H., Peterson B. M., Vestergaard M. \& Jannuzi B. T. 2005, ApJ, 629, 61

Marconi A., Hunt L. K. 2003, ApJ, 589, L21

Marconi A., Risaliti G., Gilli R., Hunt L. K., Maiolino R. \& Salvati M. 2004, MNRAS, 351, 169

Masters K. L., Springob, C. M., Haynes M. P. \& Giovanelli R. 2006, ApJ, in press (astro$\mathrm{ph} / 0609249)$

Pizzella A., Corsini E. M., Dalla Bontà E., Sarzi M., Coccato L. \& Bertola F. 2005, ApJ, 631, 785

Tremaine S. et al. 2002, ApJ, 574, 740

Yu Q., Tremaine S. 2002, MNRAS, 335, 965 